Proceedings of the 2011 Winter Simulation Conference

S. Jain, R.R. Creasey, J. Himmelspach, K.P. White, and M. Fu, eds.

\title{
SIMULATION-BASED REAL-TIME PERFORMANCE MONITORING (SIMMON): A PLATFORM FOR MANUFACTURING AND HEALTHCARE SYSTEMS
}

\author{
Alireza Mousavi \\ Brunel University \\ School of Engineering and Design \\ Electronic and Computer Engineering \\ Uxbridge, UB83PH, UK
}

\author{
Alexander Komashie \\ University of Cambridge \\ Engineering Design Centre (EDC) \\ Department of Engineering \\ Cambridge, CB2 1PZ, UK
}

\author{
Siamak Tavakoli \\ School of Electronic Engineering and Computer Science \\ Queen Mary, University of London, \\ London, E1 4NS, UK
}

\begin{abstract}
This paper introduces a new technology platform that improves the efficiency and effectiveness of simulation modelling projects. A recently developed platform that integrates data acquisition management platform (primary models) and post simulation performance analysis models (synthesis) is described. The use of real-time discrete event simulation modelers as a vehicle is proposed. In recent years we have suggested a number of solutions to integrate shopfloor data with higher level information systems. All these solutions lacked two key capabilities. Firstly, the solutions were not capable of interacting with data acquisition systems with-out expert interference in determining the quality and quantity of input signals. Therefore, connecting in-put signals to key performance indicators (i.e. simulation parameters) was extremely challenging and error prone. Secondly, from health workers' and plant managers' perspective, simulation results (e.g. resource utilization, waiting times, work-in-process, etc.) did not correspond to industry performance metrics. SIMMON is proposed here to address these two problems.
\end{abstract}

\section{INTRODUCTION}

The aim of this paper is to report on the authors' latest research results in the development of a real-time and predictive performance analysis platform in which real-time Discrete Event Simulation plays a major role. The impetus to suggest such a platform is based on the complexity of persisting and emerging problems in running modern healthcare and manufacturing systems reported by researchers and practitioners in both sectors. This complexity is being exacerbated from a myriad of new business, technological and above all social demands that affect day to day operations in typical hospitals or factories.

In two extensive literature-review papers (Komashie et al. 2007 and Jahangirian et al. 2010), the authors highlight how the process designers and analysts in healthcare sector have adopted techniques developed for manufacturing systems. The resemblances in key performance factors that determine quality of processes in both sectors are noticeable. In both industries the design of product (i.e. manufactured artifact or service required by a patient) and the sequential nature of the processes dictate the system layout and allocation of resources. Thus many of the performance measurement and optimization techniques originally designed and employed in the manufacturing industry have been acknowledged to be potentially useful for healthcare systems improvement. But effective adoption of such techniques still remains challenging for healthcare systems designers. The increasing adoption of Discrete Event Simulation in specific has been applauded by researchers and practitioners from both sectors with demonstrable 
achievements (Komashie et al. 2007, Jahangirian et al. 2010). For example, DES has helped operation managers to understand and visualize the interrelationships between processes, capacity planning and flow of material or patients (Komashie et al. 2008). Considerable research has been dedicated for developing frameworks to support healthcare managers in understanding these industry based tools and using them effectively (Jun et al. 2010). The models helped managers to improve scheduling and capacity planning, patients' flow and estimating patient waiting times with respect to system capacities.

Faced with the complexities of healthcare systems, it seems that the expectation from modelling and simulation has fundamentally changed. System analyst need to address issues that most matter to healthcare people. Secondary models such as quality of service, patient satisfaction, staff stress and overall quality of care measurements are now required.

In addition to modelling issues, the inherent shortcomings of performance measuring technologies that persist in manufacturing systems have also migrated to healthcare industry. These shortcomings do not necessarily all emanate from mathematical modelling techniques. It is difficult and at times impossible to introduce standard data acquisition and information and communication technologies (ICT) in healthcare environments.

The cost of implementing modern Supervisory Control and Data Acquisitions (SCADA) technologies are prohibitive and difficult to justify in an industry which is heavily regulated. Due to topological and architectural mismatch of legacy IT infrastructures in hospitals, any attempt to introduce new technologies within the healthcare sector faces legitimate resistance. Moreover, the human-oriented processes and complexity of patients' healthcare needs cannot easily be standardized and monitored using existing data acquisition technologies. For example, usage of active Radio Frequency Identification (RFID) cards has been refused in many institutions.

Inspired by philosophies of complexity, systems monitoring and decision making, SIMMON tries to take the first step towards an autonomous system modeler for manufacturing and healthcare systems with the above mentioned constrains in mind. Despite its internal complex structure SIMMON should make day to day performance measurement and decision making easier for line managers in both sectors. In the following sections we try to briefly explain how.

Figure 1 provides an overview of the SIMMON architecture and its building blocks.

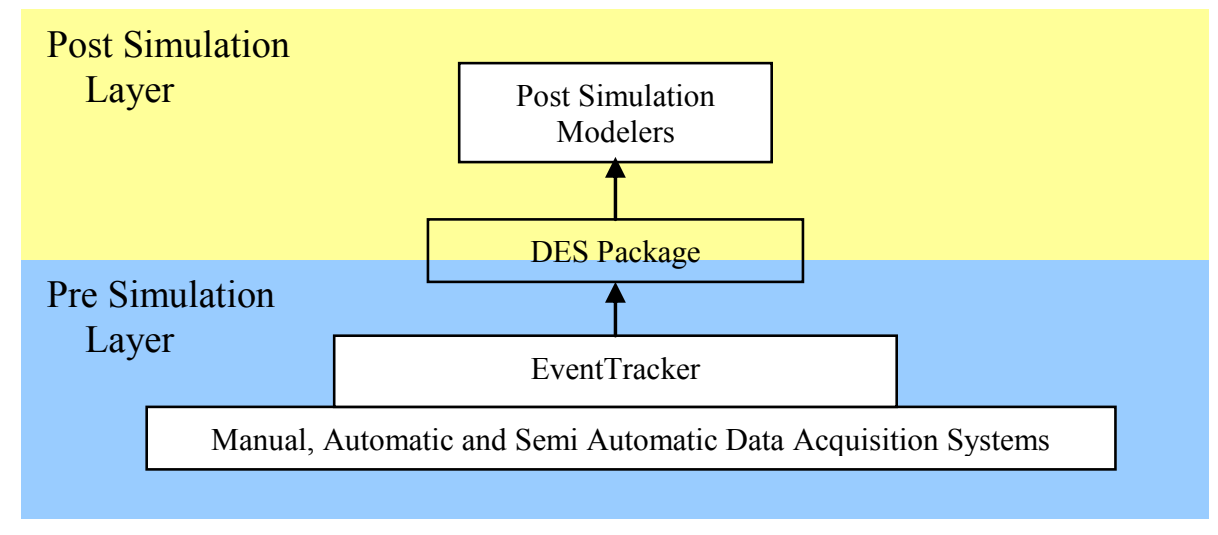

Figure 1: Overview of SIMMON Architecture

\section{THE DESIGN CONCEPT}

\subsection{Healthcare Quality Index (HQI)}

Healthcare managers are required to continuously measure, monitor and improve quality of service at the operational levels. Several approaches have been employed ranging from rigid target setting to the use of 
techniques borrowed from other industries, mainly manufacturing. In the United Kingdom's National Health Service (NHS), this agenda has been largely driven by bodies set up and with mandate from government to assess all NHS trusts. Figure 2 shows the cycle of events that such assessments have evolved in recent years.

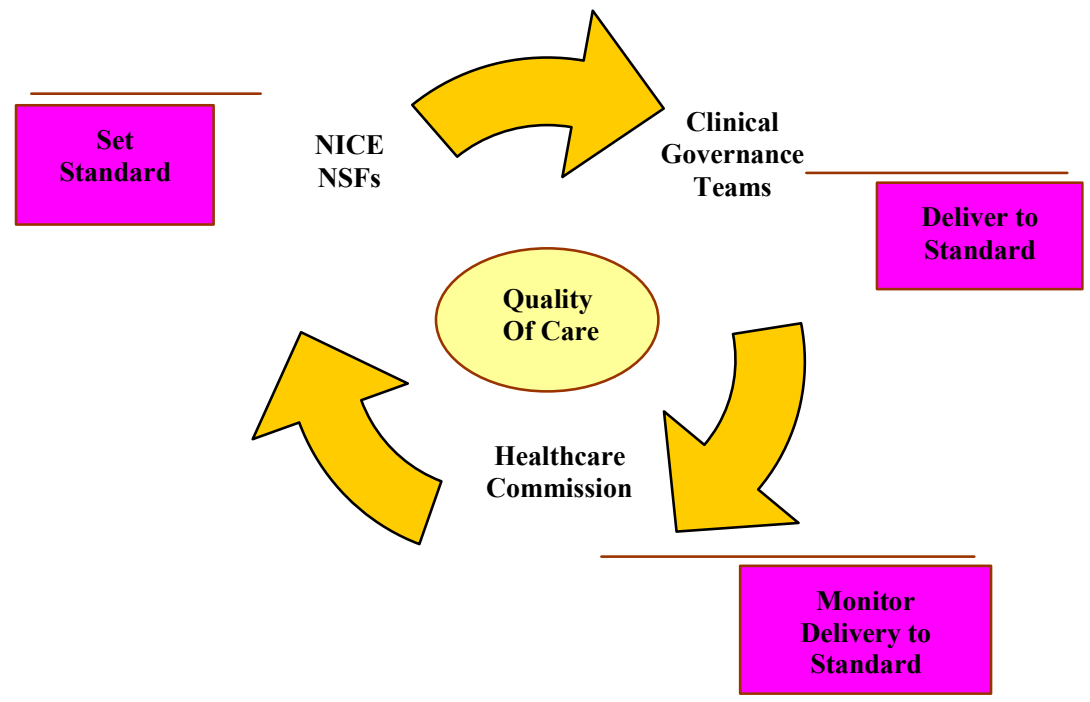

Figure 2: Cycle of performance monitoring events in the NHS

Based on this performance monitoring exercise patients and stakeholders expect the following questions to be answered:

1. Should we expect for an excellent rated Trust to provide excellent quality of service from every department in the trust?

2. Can a trust rated excellent by the survey maintain the same level of quality until the next assessment?

We believe that to confidently answer these questions, it is necessary to be able to continuously monitor and measure performance. The performance measurement should be close to real time so that corrective measures can be taken at the right time.

A simple index that would convey the level of quality from the patients' perspective to healthcare practitioners in real-time using a singular metric call Healthcare Quality Index has been introduced. The development and testing of this index is reported in (Komashie and Mousavi 2011). Using the example of an Accident and Emergency (A\&E) department, the Healthcare Quality Index (HQI) model was formulated as a linear combination of the Key Quality Indicators (KQIs) and estimated using the Generalized Maximum Entropy (GME) approach to ensure robustness (Golan et al. 1996). The formulation is presented and briefly explained in the appendix of this article.

According to Kapur and Kesavan (1992), the Lagrange's method of undetermined multipliers is the ideal tool for solving this problem. They find that because of the nature of the Shannon measure, this method helps avoid some difficult problems that are normally faced in constrained optimization. For example, when Lagrange's method is used to maximize Shannon's measure subject to linear constraints, the maximizing probabilities are all greater than zero thereby automatically satisfying the non-negativity constraint. In all constrained optimization problem, this is a difficult constraint to satisfy. 
The index was tested for robustness using Monte Carlo experiments and results compare with a Least Squares Regression (LSR) method. It was found that the GME estimated index was consistently more robust than the popular LSR method at smaller samples sizes and differing distribution of input data.

In the remaining part of this section we demonstrate how a real-time discrete event simulation system may facilitate the continuous measurement, continuous monitoring and continuous improvement of operational quality in healthcare. The use of the SIMMON framework in healthcare as a combination of the developed Event Tracker and the HQI has potential impact to significantly reduce the present time gap between measurement and improvement in the NHS as shown in figure 3.

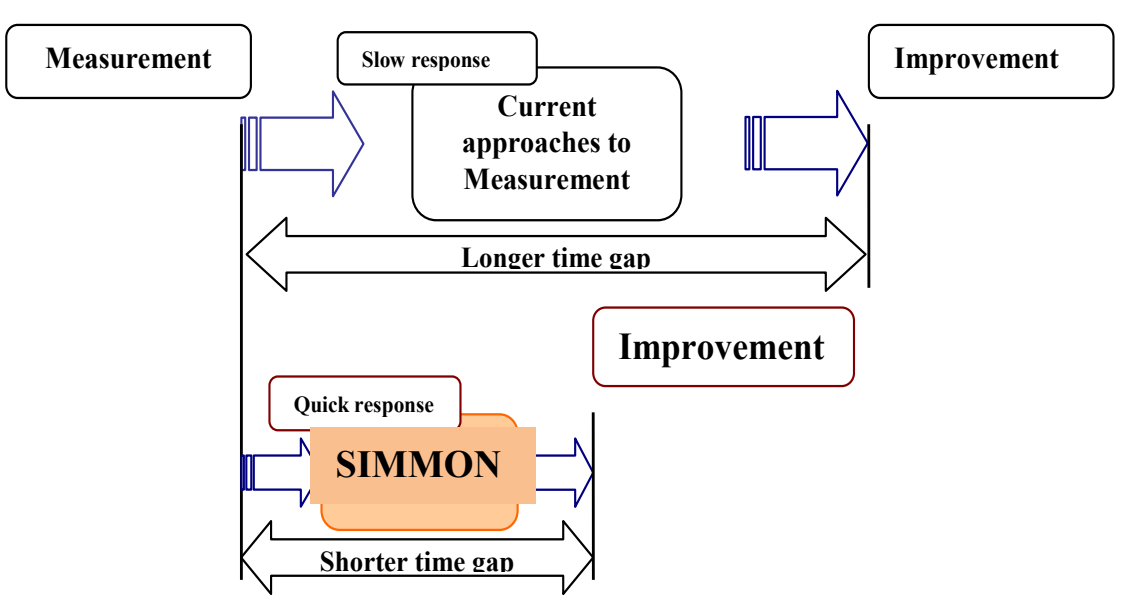

Figure 3: Proposed impact of the SIMMON framework on performance improvement

The main motivation in developing SIMMON is to increase the capabilities for continuous and meaningful system monitoring. This can not be achieved unless the importance of events is accurately measured and subsequently the quality of stochastic models determined. And all this should be conducted in timely manner (i.e. close to real-time).

\subsection{Event Tracking for Real-Time Random Behaving Systems (EventTracker)}

A continuous monitoring and performance analysis tool needs to encapsulate both human factors and technology limitations. The human factors could be cognitive, temporal, spatial or economical factors. The technology should enable seamless communication, data handling and storage thus building knowledge. On accurate knowledge, strong predictions can be made so that organization's management approach can shift from reactive fire-fighting to proactive and prudent decision making.

The proposed EventTracker (Tavakoli and Mousavi 2011) is therefore tasked to enable complex event processing in real-time. EventTracker is an event-driven sensitivity analysis method that captures and interprets events as they occur. Therefore, events are not predicted as in stochastic models but recorded when threshold has been reached (event threshold). Therefore, there is no interpretation of transition between system states. It is thus a method that does not rely on historical data or predefined probability density functions, but a deterministic occurrence.

The proposed event tracking sensitivity analysis tool uses an input-output occurrence [+, -] matrix. This matrix is designed to map the relationships between causes that trigger events (Trigger Data) and the data that describe the actual events (Event Data). By using this method EventTracker is able to construct a discrete event framework for sensitivity analysis. A description of Discrete Event System, Trigger Data, and Event Data is provided in the following subsections. 


\section{Mousavi, Komashie and Tavakoli}

\subsection{Discrete Event Systems}

As opposed to continuous system, a Discrete Event System (DES) is defined by disparate occurrence of events in a specified time span. In other words, the state of the system changes when the input variables and consequently the outputs of the system change. Each state transition of the system is called an event. Therefore, in DES, only the attributes that represent the occurrence of an event are considered. These attributes are discussed in the following section.

\subsection{Trigger Data and Event Data}

Any input variable whose value transition registers an event is defined as a Trigger Data (TD) in our DES. The series of data that represent the state of the system at a given time is described as Event Data (ED). It is possible that the numbers of EDs and TDs in a system are different. For example, a number of TD series may be responsible for changing a single ED series. However, various TD series could have differing impact on specified ED series.

$$
E D \because\left\{T D_{1}, T D_{2}, \ldots, T D_{n}\right\}
$$

This is because individual or combination of input variables may have different effects on different system outputs.

\subsection{EventTracker Method}

The algorithm implemented in the EventTracker platform is designed to respond quickly. The algorithm's life cycle is equivalent to one analysis span (AS) that is divided into several search slots (SS). Within each slot, two batches of time-bound data that were captured from two time series are used to provide a value which is translated into a sensitivity index. This is done by detection of a Trigger in trigger data (TD) batch and similarly in event data (ED) batch. Each batch is searched for fluctuations larger than a pre-specified threshold. Existence or non-existence of a change in each pair of data batches is scored as +1 ; otherwise, the score would be -1 . This index is then added to the indices of subsequent search slots. At the end of each AS, the sensitivity indices of all data series are linearly normalized. This functionality is shown in figure 4.

Table 1 shows an instant of sensitivity analysis in a specified search slot (SS).

Table 1: an example production of sensitivity index by EventTracker method.

\begin{tabular}{|c|c|c|c|c|c|c|c|c|c|c|c|}
\hline Search Slot & $\mathbf{0}$ & 1 & 2 & 3 & 4 & 5 & 6 & 7 & 8 & 9 & 10 \\
\hline Event Data & $*$ & $*$ & & $*$ & & $*$ & $*$ & $*$ & & $*$ & $*$ \\
\hline Trigger Data 1 & & & $*$ & & & $*$ & & & $*$ & $*$ & $*$ \\
\hline Sensor 1 & -1 & -1 & -1 & -1 & 1 & 1 & -1 & -1 & $-\quad 1$ & 1 & 1 \\
\hline Sensitivity Index 1 & -1 & -2 & -3 & -4 & -3 & -2 & -3 & -4 & -5 & -4 & -3 \\
\hline $\begin{array}{l}\text { Normalized Sensitivi- } \\
\text { ty Index } 1\end{array}$ & 0.00 & 0.00 & 0.00 & 0.00 & 0.00 & 0.00 & 0.00 & 0.00 & 0.00 & 0.00 & 0.00 \\
\hline Trigger Data 2 & $*$ & & & & $*$ & $*$ & $*$ & $*$ & & $*$ & \\
\hline Sensor 2 & 1 & -1 & 1 & -1 & -1 & 1 & 1 & 1 & 1 & 1 & -1 \\
\hline Sensitivity Index 2 & 1 & 0 & 1 & 0 & -1 & 0 & 1 & 2 & 3 & 4 & 3 \\
\hline $\begin{array}{l}\text { Normalized Sensitivi- } \\
\text { ty Index } 2\end{array}$ & 1.00 & 1.00 & 1.00 & 0.67 & 0.33 & 0.33 & 0.67 & 0.75 & 0.80 & 0.80 & 0.75 \\
\hline Trigger Data 3 & & $*$ & & $*$ & & $*$ & & $*$ & & $*$ & \\
\hline Sensor 3 & -1 & 1 & 1 & 1 & 1 & 1 & -1 & 1 & 1 & 1 & -1 \\
\hline Sensitivity Index 3 & -1 & 0 & 1 & 2 & 3 & 4 & 3 & 4 & 5 & 6 & 5 \\
\hline $\begin{array}{l}\text { Normalized Sensitivi- } \\
\text { ty Index } 3\end{array}$ & 0.00 & 1.00 & 1.00 & 1.00 & 1.00 & 1.00 & 1.00 & 1.00 & 1.00 & 1.00 & 1.00 \\
\hline
\end{tabular}




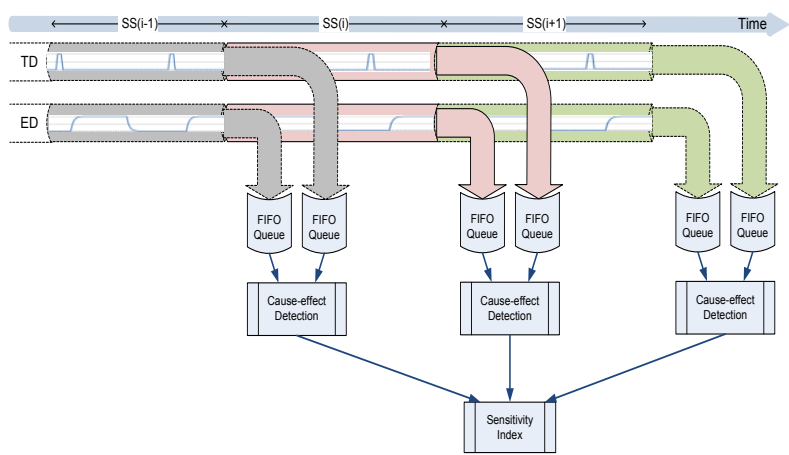

Figure 4: Overview of EventTracker method

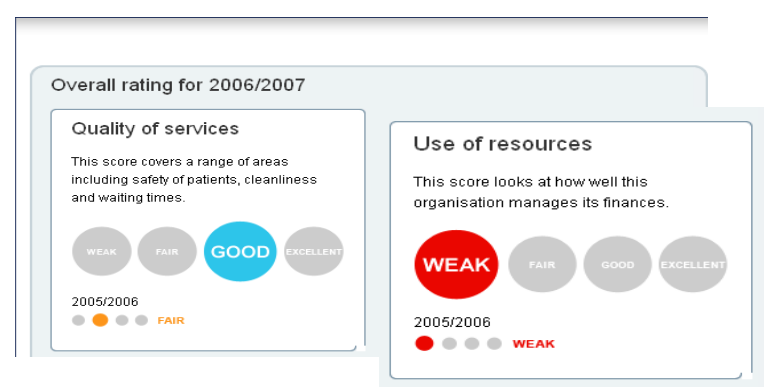

Figure 5: A snap shot from the Healthcare Commission's 2006/2007 assessment results

The output of the EventTracker is then feed into a simulation model in which the data provides the key input variables for measuring simulation output such as availability of resources, capacity fluctuations, resource utilization, flow of material, time between arrivals,... . The output of the simulation model is then fed into secondary models that produce singular cost or quality of service parameters.

\section{CASE STUDIES}

\subsection{Healthcare}

Figure 5 shows a snapshot from the 2006/2007 Healthcare Commission's assessment results of an NHS Trust. It can be seen that the trust scored 'good' for quality of services and 'weak' for use of financial resources.

The current system is unable to guarantee the measured level of quality consistently throughout all departments of the trust and until another assessment a year after. This is the gap that SIMMON for Healthcare seeks to fill. We believe that healthcare practitioners are more able to respond effective to improvement challenges when the information they need is timely.

We take an emergency department for example where figure 6 shows the physical set up of the system, the Discrete Event Simulation of the system, the Event Tracker and the Real-time performance indicator display. The system shown here does not include the real-time survey analysis feature. That has been demonstrated separately and has not yet been integrated into the entire framework. The indicators displayed are therefore based on random mathematical formulations. 


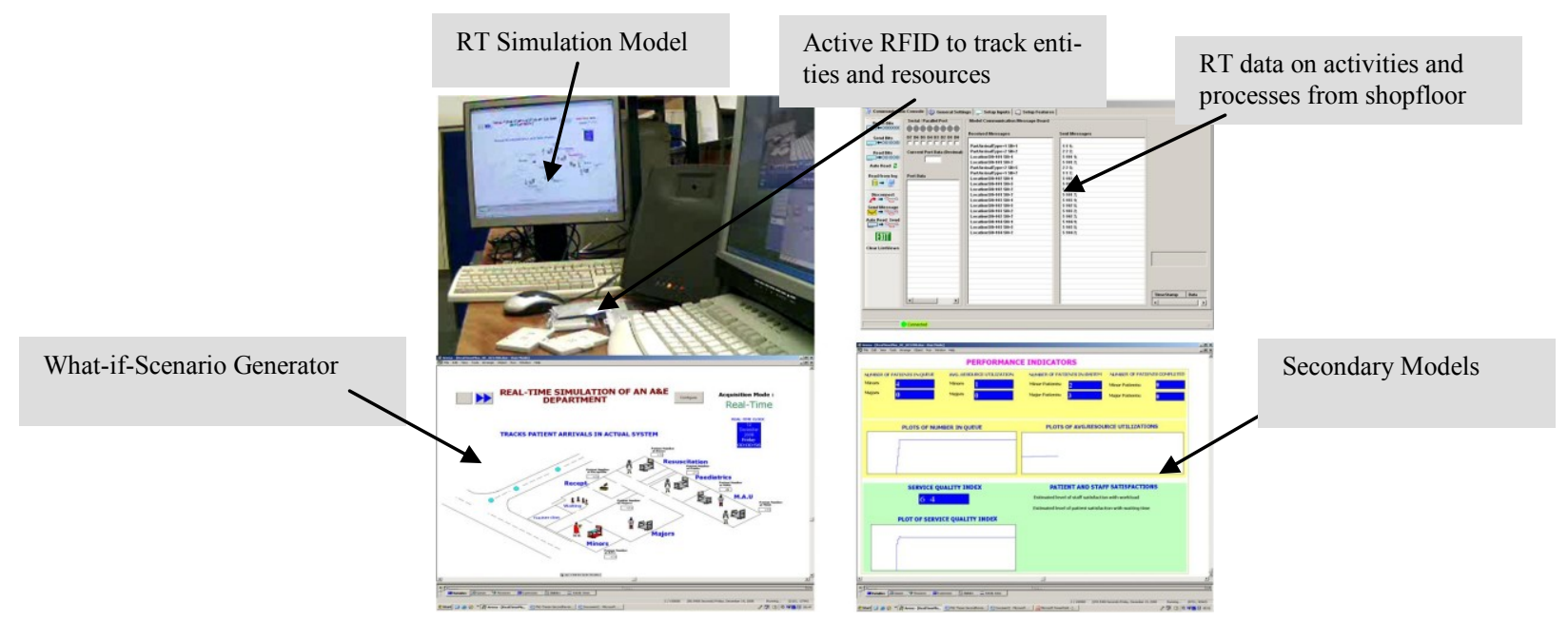

Figure 6: Components of the SIMMON platform for Healthcare

The simulation model was integrated to the EventTracker through the designed and developed Flexible Data Input Layer Architecture (Tavakoli et al. 2008). In order to detect arrivals of patients and trace their journey through the system, the R3M solution proposed in Tavakoli et al (2008) integrates the Radio Frequency Identification (RFID) technology with Real-Time Arena software tool. This example was enacted in a laboratory environment. The implications of this platform for healthcare service improvement are discussed in section 4.

\subsection{Manufacturing}

A real-time discrete-event model of the production process of a refrigerator manufacturing plant was developed to help with measuring a number of production performance parameters. The production process in conjunction with complete mapping of the sensors and actuators on the production line are shown in figures 7-9. Each diagram shows a process and its constituent operations. It also shows signals that trigger the start and completion of each task (i.e. an event). The parameter chosen for production performance analysis is the Instantaneous Resource Utilization.

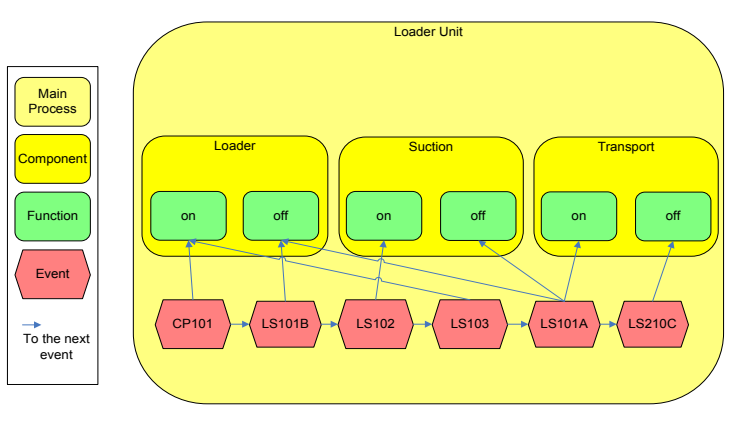

Figure 7: Loader processes and events

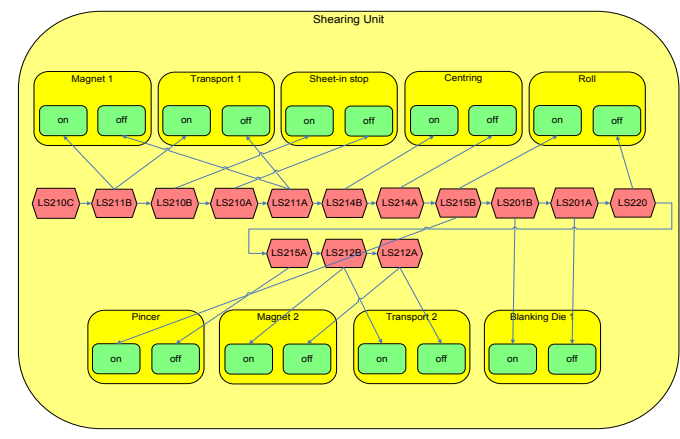

Figure 8: Shearing Unit processes and events 


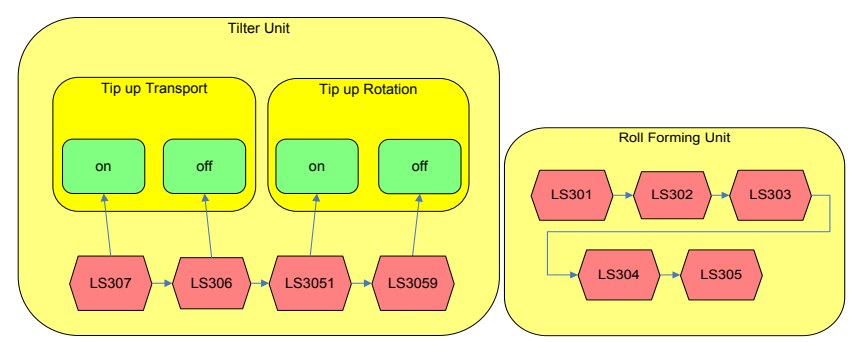

Figure 9: Tilter Unit and Roll Forming Unit processes and events

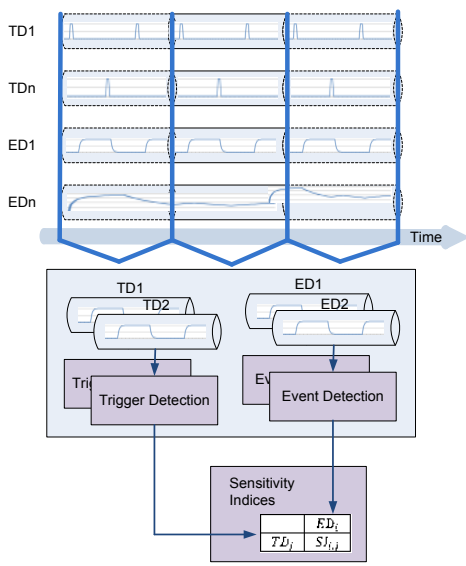

Figure 10: Implementation of EventTracker for multiple Input Variables and Output Parameters

There were 28 input variables streamed from the shopfloor generated by sensors installed on the production line. The 28 TD input values were linked to four event data (ED) series. The data was fed into the EventTracker modeler at the same rate as they were collected during a period of 2 minutes (i.e. 500 data points at 5 samples per second). This integration between EventTracker and Arena software tool allowed for direct translation of multiple signals to instantaneous resource utilization values of 4 machines. The R3M mechanism described by Tavakoli et al. (2008) was implemented so that the relationship between signals and processes was well-defined within the structure of the model.

The implementation of EventTracker on the production line is shown in Figure 10. As a result, a two dimensional array of sensitivity indices in the time domain was generated.

The key feature of the proposed method is the rapid filtering of unimportant data that may very well at times overwhelm the data processing platforms. It is safe to claim that with regard to the time domain, the EventTracker method may be classified as a Local Sensitivity Analysis (LSA) method. Moreover, to estimate sensitivity indices, the EventTracker method does not require any prior knowledge about the analytical relationship between input and output variables. The EventTracker, in this sense, can be considered as a Global Sensitivity Analysis method. In this sense will not only be able to link the input data and performance (e.g. resource utilization) variables but also determine the relationship between the input data and the performance variable. This would allow a system designer to only install/connect sensors and actuators that are important to the Discrete Event Simulation models thus reducing the communication and computing load.

\section{THE IMPACT AND IMPLICATIONS FOR SYSTEM IMPROVEMENT}

The automation of input data collection and determining the interdependencies between input data is the most challenging part of any discrete event simulation modelling project. The proposed solution in tracking and linking events as they occur will improve the efficiency of pre-modelling activities in any simulation project.

In our experience with traditional simulation project, depending on the availability of input data in the correct format the data collection and interpretation process could take up to $70 \%$ of the total project time. Installation of SIMMON can save the time and cost of typical simulation projects by $80 \%$. The sensitivity analysis proposed in this architecture will increase the accuracy and quality of input data whilst reducing redundancy. In a recent project in a refrigerator manufacturing plant we successfully identified 7 out of 28 sensors to be redundant and by removing them from the panel production line, the sensitivity analysis was conducted in a fraction of the time it was conducted with original setting and using a different sensitivity analysis technique (Tavakoli and Mousavi 2011). 


\section{Mousavi, Komashie and Tavakoli}

We believe that this technique will be even more applicable to labor intensive environments such as Healthcare systems where human factors could cause major complexities and disruption to operations. A future plan is to deploy SIMMON into a real hospital or medical centre department.

On Synthesis of Simulation Output Results, the proposed SIMMON platform for healthcare has the potential of contributing significantly to several of the improvement challenges faced in the healthcare system of the United Kingdom. These challenges have cast doubts on current methods of quality measurement and monitoring through annual assessments in some cases. For instance, Mayor (2003) conducted a survey of $100 \mathrm{~A} \& \mathrm{E}$ consultants and found that $56 \%$ used temporary medical and nursing staff to reduce patient waiting times during quality monitoring weeks, in which $25 \%$ reported that they allow staff to work double hours during this period. These challenges have been considered as the result of ignoring the underlying systemic nature of the healthcare environment over the past several years (Umble and Umble 2005). These problems indicate the need for further research into effective measurement, monitoring and improvement of healthcare quality (Bird et al 2005).

As early as 1966, Donabedian outlined the necessity of measuring healthcare quality using indices. Since then researchers and practitioners have fallen short of offering a universally acceptable healthcare index that enables system experts to continuously provide a measure of quality of care.

We believe that the introduction of SIMMON may pave the way to addressing this need. The monitoring and healthcare quality measurement capabilities provided by SIMMON can transform healthcare management from fire fighting mentality into proactive response to factors that impede quality of care.

\section{CONCLUSIONS}

In this paper the authors reported on their experience with traditional simulation modelling projects and discussed the key shortcomings in terms of efficiency and effectiveness. At times we realized that $70 \%$ of the effort and cost of a project consumed by input data collection and processing. Moreover once the simulation project was successfully completed, the simulation results were normally complicated and incomprehensible for our clients. It is well noted that researchers and practitioners in both manufacturing and healthcare industries now believe that traditional simulation is capable of identifying operational malaise and offer what-if-scenario alternatives to improve operations at no cost or disruption to the actual system. However, they have realized that traditional simulation alone is incapable of measuring the impact of simulation outputs in the form of simple more inclusive business performance parameters such as: Quality of Service, customer/patient satisfaction, cost (Mousavi et al. 2007) or environmental impact. The missing link between simulation output and universally understood business performance parameters has contributed to the perceived ineffectiveness of traditional simulation modelling projects.

The recently developed SIMMON solution proposed in this paper is one of the most effective technologies to deal with the efficiency and effectiveness of simulation modelling. It allows for a myriad data acquisition technologies to be connected to an input data processing unit that prepares the information for Real-Time Simulation package. It will then translate the simulation results into comprehensible business performance parameters, thus integrating engineering, mathematical modelling and management principles in one. The main savings time and computational effort in data acquisition efforts data modelling and analysis was reported in (Tavakoli et al. 2008 and 2011). The effectiveness and impact of the modelling solutions in specific in healthcare industry have been reported in (Komashie et al. 2008 and 2011).

Two case studies in the healthcare and manufacturing industry have been reported. The proposed platform is at its proof of concept stage. In order to further validate and verify the proposed technology and models further tests are required.

\section{REFERENCES}

Bevan G. and C. Hood. 2004. "Targets, inspections, and transparency" British Medical Journal, 328, 7440: 598. 
Bird, S. M., D. Cox, T. Farewell, H. Goldstein, T. Holt, and P. Smith. 2005. "Performance indicators: good, bad, and ugly", Journal of the Royal Statistical Society, 168, 1: 1-27.

Golan, A., G. Judge and L. Karp. 1996. "A maximum entropy approach to estimation and inference in dynamic models or counting fish in the sea using maximum entropy," Journal of Economic Dynamics and Control, Vol. 20, No. 4, 559-582.

Jahangirian, M., T. Eldabi, A. Naseer, L. Stergioulas, and T. Young. 2010. "Simulation in manufacturing and business: a review" European Journal of Operational Research, 203, 1:1- 13.

Jun, G., S. Hinrichs, T. Jafri, and P. Clarkson. 2010. "Thinking with simple diagrams in healthcare systems design", 11th International Design Conference (DESIGN 2010), Dubrovnik, Croatia.

Jun, G., J. Ward and P. Clarkson. 2010. "Systems modelling approaches to the design of safe healthcare delivery: ease of use and usefulness perceived by healthcare workers", Ergonomics, 53, 7: 829-847.

Jun, G., J. Ward, Z. Morris and P. Clarkson. 2009. "Health care process modelling: which method when?" International Journal for Quality in Health Care, 21, 3: 214-224.

Kapur, J. and Kesavan, H. 1992. Entropy Optimization Principles with Applications. Academic Press Inc.

Komashie A., A. Mousavi and J. Gore. 2007. "Quality management techniques in healthcare and manufacturing industry: A comparative review and emerging issues" Journal of Management History, 13, 4: 359-370.

Komashie, A., A. Mousavi and J. Gore. 2008. "Using Discrete Event Simulation to Manage Theatre Operations in Healthcare: An Audit-Based Case Study" Proceeding of 2008 IEEE EUROSIM/UKSim'08 Conference, Cambridge, UK.

Komashie, A., A. Mousavi. 2011. "Measuring the Quality of healthcare: A Robust Healthcare Quality Index (HQI) based on the Generalised Maximum Entropy Formulation", forthcoming.

Mayor, S. 2003. "Hospitals take short term measures to meet targets," British Medical Journal, 326, 7399:1054.

Mousavi A., Hamdi, M. and Sarhadi M. 2007. "Knowledge-Based Quick-Response Decision Making in the Food Processing Industry," International Journal of Industrial Systems Engineering, Vol. 2, No.1, 99-122.

Tavakoli, S., A. Mousavi and A. Komashie. 2008. "A Generic Framework for Real-Time Discrete Event Simulation (DES) Modelling." In Proceedings of 2008 Winter Simulation Conference, edited by S. J. Mason, R. R. Hill, L. Mönch, O. Rose, T. Jefferson, J. W. Fowler, 1931-1938. Piscataway, New Jersey: Institute of Electrical and Electronics Engineers, Inc.

Tavakoli, S., A. Mousavi and A. Komashie. 2008, "Flexible data input layer architecture (FDILA) for quick-response decision making tools in volatile manufacturing systems2," In Proceedings of 2008 IEEE International Conference on Communications, Beijing, 5515-5520.

Tavakoli, S. and A. Mousavi. 2011. "Event Tracking for Real-Time Unaware sensitivity Analysis", IEEE Transactions in Knowledge and Data Engineering, in print.

Umble, M. and E. Umble. 2006. "Utilizing buffer management to improve performance in a healthcare environment" European Journal of Operational Research, 174, 2:1060-1075.

Wolstenholme E, D. Monk, G. Smith and D. McKelvie. 2005. "Coping but not Coping in Health and Social Care - masking the reality of running organizations well beyond safe design capacity", International System Dynamics Society Conference; 2005; Boston, USA.

Donabedian, A. 1966. "Evaluating the quality of medical care." The Milbank Memorial Fund Quarterly, Vol. 44, No.3, Pt. 2,166-203.

\section{Appendix}

$$
I=\gamma_{1} y_{1}+\gamma_{2} y_{2}+\gamma_{3} y_{3}+\ldots+\gamma_{j} y_{j}+\ldots+\gamma_{n} y_{n}
$$

where $\gamma_{j}$ is the weight of the KQI (Key Quality Indicator) $\mathrm{y}_{j}$ 
We further assume that the KQIs are each a linear combination of their respective factor variables given by a multiple regression model as:

$$
\begin{aligned}
& y_{1}=\beta_{11} x_{11}+\beta_{12} x_{12}+\beta_{13} x_{13}+\ldots+\beta_{1 i} x_{1 i}+\ldots+\beta_{1 m} x_{1 m}+\varepsilon_{1} \\
& y_{2}=\beta_{21} x_{21}+\beta_{22} x_{22}+\beta_{23} x_{23}+\ldots+\beta_{2 i} x_{2 i}+\ldots+\beta_{2 m} x_{2 m}+\varepsilon_{2} \\
& \begin{array}{llllll}
\vdots & \vdots & \vdots & \vdots & \vdots & \vdots
\end{array} \\
& y_{j}=\beta_{j 1} x_{j 1}+\beta_{j 2} x_{j 2}+\beta_{j 3} x_{j 3}+\ldots+\beta_{j i} x_{j i}+\ldots+\beta_{j m} x_{j m}+\varepsilon_{j} \\
& y_{n}=\beta_{n 1} x_{n 1}+\beta_{n 2} x_{n 2}+\beta_{n 3} x_{n 3}+\ldots+\beta_{n i} x_{n i}+\ldots+\beta_{n m} x_{n m}+\varepsilon_{n}
\end{aligned}
$$

where

$\beta_{j i}$ is a regression coefficient and $x_{j i}$ is a satisfaction value for a sub factor $i$ under KQI $\mathrm{y}_{j}$ In Matrix form, we have; $\mathbf{H}=\mathbf{X} \cdot \mathbf{B}+\mathbf{E}$

Where: $\mathbf{H}$ is an $n \times 1$ column vector of KQI values; $\mathbf{B}$ is an $m \times 1$ column vector of regression coefficients; $\mathbf{X}$ is an $n \times m$ matrix of sub factor values; and $\mathbf{E}$ is an $n \times 1$ vector of residual errors in the regression equations. The unknown parameters and disturbance terms need to be re-parameterized according to the requirements of the GME estimation principles. The matrix equation above therefore becomes;

\section{$\mathbf{H}=\mathbf{X} \cdot \mathbf{Z} \cdot \mathbf{p}+\mathbf{V} \cdot \mathbf{w}$}

where $\mathbf{H}$ is an $n \times 1$ column vector of KQI values; $\mathbf{X}$ is a $n \times m$ matrix of sub factor values; and $\mathbf{Z}$ is an $m \times m$ diagonal matrix of support variables for the coefficients. Each element of $\mathbf{Z}$ is a row vector

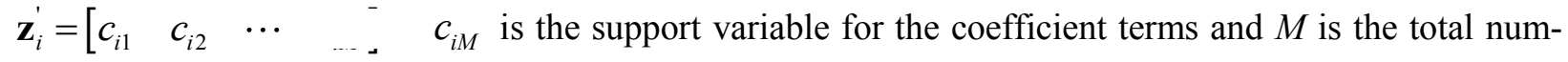
ber of support points in $\mathbf{z}_{i}^{\prime} . \mathbf{V}$ is an $n \times n$ diagonal matrix of support variables for the residual terms. Each element of $\mathbf{V}$ is a row vector: $\mathbf{v}_{j}^{\prime}=\left[\begin{array}{lllllll}b_{j 1} & b_{j 2} & \cdots & \neg & \ldots & . & b_{j N}\end{array}\right.$ is the support variable for the residual terms and $N$ is the total number of support points in $\mathbf{v}_{j}^{\prime} \cdot \mathbf{p}$ and $\mathbf{W}$ are probability vectors associated with the coefficients and random errors. That is;

$\mathbf{p}=\left[\begin{array}{l}\mathrm{p}_{1} \\ \mathrm{p}_{2} \\ \vdots \\ \mathbf{p}_{i} \\ \vdots \\ \mathrm{p}_{m}\end{array}\right]$ and $\mathbf{p}_{i}=\left[\begin{array}{c}\mathrm{p}_{i 1} \\ \mathrm{p}_{i 2} \\ \vdots \\ \mathrm{p}_{i . M}\end{array}\right]$

$\mathbf{w}=\left[\begin{array}{l}\mathrm{w}_{1} \\ \mathrm{w}_{2} \\ \vdots \\ \mathbf{w}_{j} \\ \vdots \\ \mathrm{w}_{n}\end{array} \mid \quad\right.$ and $\mathbf{w}_{j}=\left[\begin{array}{c}\mathrm{w}_{j 1} \\ \mathrm{w}_{j 2} \\ \vdots \\ \mathrm{w}_{j . N}\end{array}\right]$

Therefore the coefficients and error terms will be given by: 


$$
\begin{aligned}
& \left.\boldsymbol{\beta}=\mathbf{Z} \cdot \mathbf{p}=\left[\begin{array}{cccccc}
\mathbf{z}_{1}^{\prime} & 0 & \cdots & & 0 \\
0 & \mathbf{z}_{2}^{\prime} & \cdots & & 0 \\
\vdots & \vdots & \ddots & & \vdots & \vdots \\
0 & 0 & 0 & \ddots & & \mid \\
0 & 0 & \cdots & & i_{m}^{\prime}
\end{array}\right] \mid \begin{array}{c}
\mathbf{p}_{1} \\
\mathbf{p}_{2} \\
\mid \mathbf{p}_{m}
\end{array}\right] \\
& \boldsymbol{\varepsilon}=\mathbf{V} \cdot \mathbf{W}=\left[\begin{array}{cccccc}
\mathbf{v}_{1}^{\prime} & 0 & \cdots & & 0 \\
0 & \mathbf{v}_{2}^{\prime} & \ldots & & 0 \\
\vdots & \vdots & \ddots & & \vdots & \text { । । } \\
0 & 0 & 0 & \ddots & & \vdots \\
0 & 0 & \ldots & & i_{n}^{\prime}
\end{array}\right]\left\lfloor\left[\begin{array}{c}
\mathbf{w}_{1} \\
\mathbf{w}_{n}
\end{array}\right]\right.
\end{aligned}
$$

The whole equation then becomes;

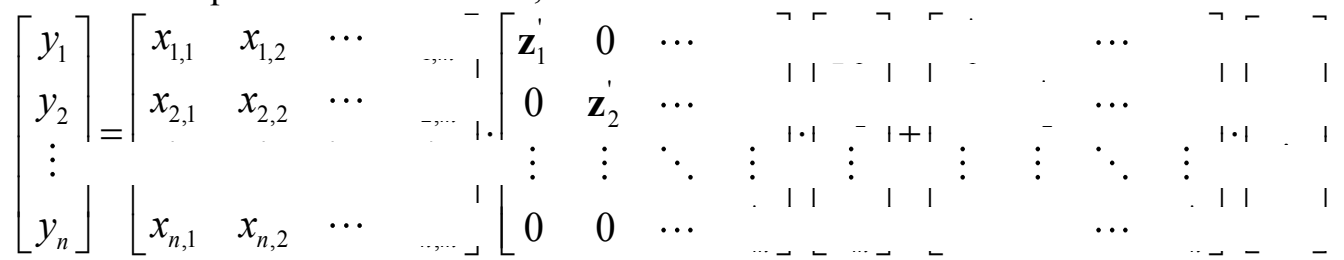

With the re-parameterized version, the GME equation can now be expressed as a non-linear programming problem subject to linear constraints. Thus maximizing: $H(p, w)=-\mathbf{p}_{1, M}^{\prime} \ln \mathbf{p}_{M, 1}-\mathbf{w}_{1, N}^{\prime} \ln \mathbf{w}_{N, 1}$

Subject to the data consistency constraint and the adding-up constraints:

$$
\sum_{m=1}^{M} p_{i m}=1 \quad \sum_{n=1}^{N} w_{j n}=1
$$

\section{AUTHOR BIOGRAPHIES}

ALIREZA MOUSAVI is senior lecturer of Systems Engineering and Computing in Brunel University, UK. His research interest is in Mathematical Modelling and Simulation, Applied Control and Computing.

ALEXANDER KOMASHIE is a research associate at the Engineer and Design Centre, Cambridge University, UK. His $\mathrm{PhD}$ thesis titled "Information-Theoretic and Stochastic Methods for Managing the Quality of Service and Satisfaction in Healthcare Systems" proposed the "E-Track NHS" system which looked at the application of real-time Discrete Event Simulation for improving healthcare system performance from Brunel University, UK. He therefore has a keen interest in the use of the systems approach and particularly the modelling and simulation of healthcare systems.

SIAMAK TAVAKOLI completed his PhD in real-time systems modelling from the School of Engineering and Design at Brunel University. He received a B.Sc. in Electronic Engineering from Amirkabir University of Technology in Tehran. His current research interests include real-time data acquisition and simulation for decision making tools. Siamak is a member of IEEE, Robotics and Automation Society, and Industrial Electronics Society. He is currently a research associate in the University of Queen Mary London, UK. 